Wagner: Eine Modification des Knop'schen Azotometers.

FerrocyankaliumMethode.

\begin{tabular}{|c|c|c|c|c|c|c|}
\hline Reiches & Kupfererz & (Stufferz.) & 6,94 & oCt. & 7,09 & pCt. \\
\hline Ordinäres & $8 \quad \ll$ & (Stufferz.) & 3,06 & $\ll$ & 3,18 & $\ll$ \\
\hline$\ll$ & $\ll$ & (Bergkern.) & 3,57 & $\ll$ & 3,90 & * \\
\hline Armes & $\ll$ & (Stufferz.) & 3,90 & $\ll$ & 3,80 & $\ll$ \\
\hline Melirtes & Erz & (Stufferz.) & 10,19 & « & 9,98 & $\ll$ \\
\hline$\ll$ & * & (Bergkern.) & 17,72 & $\ll$ & 17,34 & $\ll$ \\
\hline$\ll$ & $\ll$ & (Gräupel.) & 12,64 & 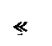 & 12,94 & $\ll$ \\
\hline Bleierze & & (Stufferz.) & 22,70 & $\ll$ & 22,92 & « \\
\hline$\ll$ & & (Waschkern.) & 20,01 & $\ll$ & 20,31 & $\ll$ \\
\hline$\ll$ & & (Gräupel.) & 19,77 & $y$ & 19,28 & $\ll$ \\
\hline$\ll$ & & (Schlich.) & 17,35 & $\ll$ & 17,74 & $\ll$ \\
\hline
\end{tabular}

Ich glaube, dass die Titration mit Ferrocyankalium manche Vorzüge vor den bis jetzt bekannten maassanalytisehen Methoden hat. Sie bietet, was Schnelligkeit und Genauigkeit anbetrifft, manche Bequemlichkeit für den, der des Tags 10-15 Bestimmungen ausführen soll, und wenn man bei allen Nachtheilen mit denen die Schwefelnatrium-Methode schon behaftet ist noch bedenkt, wie lästig die Darstellung der Schwefelnatriumlösung, so greift man jedenfalls lieber zum Ferrocyankalium, das als chemisch reine Handelswaare aus einer jeden chemischen Fabrik leicht $\mathrm{zu}$ beziehen ist.

Eine Modification des Knop'schen Azotometers. Von

\title{
Dr. Paul Wagner.
}

Die volumetrische Stickstoff bestimmung unter Anwendung des von Professor K nop construirten Azotometers ist wegen ihrer bequemen Ausführung nicht nur, sondern auch wegen ihrer Zuverlässigkeit and Genauigkeit eine der vorzüglichsten analytischen Methoden.

Gelegentlich einer Arbeit über Bodenabsorption wurde ich veranlasst, die Branchbarkeit des Knop'schen Azotometers unter Berücksichtigung der von Dietrich*) gegebenen Bemerkungen sorgfältig zu prüfen und

*) Siehe diese Zeitschrift 5, 36 . 
fand dabei, dass einige Modificationen in Construktion und Gebrauch des Apparates zur Vervollkommnung desselben vorzunehmen waren.

Die von mir angebrachten Aenderungen, deren Zweckmässigkeit ich durch längeren Gebrauch des Apparates und an einer sehr grossen Reihe von Untersuchungen genügend erprobt habe, theile ich nachstehend mit.

Als bekannt setze ich die frühere Construktion und die Prüfung des Knop'schen Azotometers voraus, verweise eventuell auf die betreffende Abhandlung von Prof. Knop*) and eine Abbildung des Apparates in Fresenius' Anleitung zur quant. Analyse 5. Aufl. S. 877.

Knop wandte zuerst bromirte Javell e'sche Lauge als Zersetzungsflüssigkeit an, fand aber, dass diese Lösung nur bei Entwickelung von $10-25 \mathrm{CC}$. Stickstoff brauchbar war, nicht aber, wenn grössere Mengen von Ammoniak zersetzt werden sollen und hat für den letzteren Fall eịne Lösung von unterbromigsaurem Baryt oder unterbromigsaurem Natron vorgeschlagen. Ich habe gefunden, dass die letztere Flüssigkeit, die nach K n op'scher Vorschrift dargestellte Lösung von unterbromigsaurem Natron, in allen Fällen die besten Resultate liefert. Die Zersetzung des Ammoniaks geht bei Anwendung dieser Lösung schnell und vollständig von Statten, während die bromirte Javelle'sche Lauge nur so weit zufriedenstellende Resultate liefert, als das in ihr enthaltene B rom hinreicht, um die Zersetzung zu bewirken.

Der in der Zersetzungsflüssigkeit gelöst bleibende Theil Stickstoff ist von $\mathrm{Knop}$ nicht in Rechnung gebracht worden, da, wie $\mathrm{Knop}$ angibt, der hieraus entspringende Feller nur gering sei, und auch bei grösserer Verdünnung der Lauge (welche in concentrirtem Zustandekeinen Stickstoff zurückhält) nur unerheblich zunehme. Entwickele man 15-50 CC. Stickstoff, so könne man mit ein nnd demselben Quantum. Lauge $6-7$ Bestimmungen hintereinander machen und prüfen, ob bei der ersten ein Fehler durch Absorption eingetreten sei oder nicht. Ich habe dies vielfach geprüft und stets eine mit der Verdünnung proportionale und nicht unerhebliche Zunahme dieses Fehlers gefunden.

Folgende zwei Versuchsreihen mögen als Beispiele dienen:

I.

4,005 Grm. salpetersaures Ammoniak wurden in Wasser gelöst und. auf $500 \mathrm{CC}$. verdünnt. $20 \mathrm{CC}$. dieser Lösung wurden jedesmal durch

*) Diese Zeitschrift 9, 225 und Chem. Centralbl. 1860, 244. 
200 CC. Bromlange zersetzt, indem zum ersten Versuch die unverdünnte, zum zweiten die beim ersten gebrauchte, zum dritten die beim zweiten Versuch gebrauchte Bromlauge u. s. w. angewendet wurde.

1) 20 CC: ergaben 24,0 CC. Stickstoff.

Lafttemperatur $16^{\circ} \mathrm{C}$. Barometerstand $758^{\mathrm{mm}}$

$1 \mathrm{CC} .=1,16282 \mathrm{Mgr}$.

$24,0 \times 1,16282=27,91 \mathrm{Mgr} .$, hieraus Procent 17,42.

Rechnung $28,03 \ll \ll 17,50$.

2) 20 CC. ergaben 23,7 CC. Stickstoff.

Luftemperatur $16^{0} \mathrm{C}$. Barometerstand $758^{\mathrm{mm}}$

1 CC. $=1,16282 \mathrm{Mgr}$.

$23,7 \times 1,16282=27,56 \mathrm{Mgr} .$, hieraus Procent $17,20$.

Rechnung $28,03 \ll \quad \ll \quad 17,50$.

3) 20 CC. ergaben 23,6 CC. Stickstoff.

Luftemperatur $16^{0} \mathrm{C}$. Barometerstand $758^{\mathrm{mm}}$

$1 \mathrm{CC} .=1,16282 \mathrm{Mgr}$.

$23,6 \times 1,16282=27,43 \mathrm{Mgr}$., hieraus Procent 17,11 .

Rechnung $28,03 \ll \ll 17,50$.

4) 20 CC. ergaben 23,6 CC. Stickstoff.

Lafttemperatur $170 \mathrm{C}$. Barometerstand $758^{\mathrm{mm}}$

$1 \mathrm{CC} .=1,15741 \mathrm{Mgr}$.

$23,6 \times 1,15741=27,31 \mathrm{Mgr}$., hieraus Procent 17,04 .

Rechnung $28,03 \quad \ll \quad \ll \quad 17,50$.

5) 20 CC. ergaben 23,6 CC. Stickstoff.

Lufttemperatur $17^{0} \mathrm{C}$. Barometerstand $758^{\mathrm{mm}}$

1 CC. $=1,15741 \mathrm{Mgr}$.

$23,6 \times 1,15741=27,31 \mathrm{Mgr} .$, hieraus Procent $17,04$.

Rechnung $28,03 \quad \ll \quad \ll 17,50$.

6) 20 CC. ergaben 23,4 CC. Stickstoff.

Lufttemperatur $17^{\circ}$ C. Barometerstand $758 \mathrm{~mm}$

1 CC. $=1,15741 \mathrm{Mgr}$.

$23,4 \times 1,15741=27,08 \mathrm{Mgr}$., hieraus Procent 16,90.

Rechnung $28,03 * \quad * \quad * 17,50$.

II.

4,510 Grm. Chlorammonium wurden in Wasser gelöst, auf $500 \mathrm{CC}$. verdünnt und in derselben Weise wie bei $\mathrm{I}$. die folgenden Versuche damit angestellt: 
Wagner: Eine Modification des Knop'schen Azotometers.

1) 20 CC. ergaben 40,5 CC. Stickstoff.

Lufttemperatur $15^{\circ} \mathrm{C}$. Barometerstand $754^{\mathrm{mm}}$.

$1 \mathrm{CC} .=1,16187 \mathrm{Mgr}$.

$40,5 \times 1,16187=47,06 \mathrm{Mgr}$., hieraus Procent 26,086.

Rechnung $47,24 \quad * \quad \ll 26,186$.

2) 20 CC. ergaben 40,1 CC. Stickstoff.

Lufttemperatur $15^{0}$ C. Barometerstand $754^{\mathrm{mm}}$.

$1 \mathrm{CC} .=1,16187 \mathrm{Mgr}$.

$40,1 \times 1,16187=46,59$ Mgr., hieraus Procent 25,826.

Rechnung $47,24 \quad \ll \quad \ll 26,186$.

3) 20 CC. ergaben 39,9 CC. Stickstoff.

Lufttemperatur $15^{0}$ C. Barometerstand $754^{\mathrm{mm}}$.

1 CC. $=1,16187 \mathrm{Mgr}$.

$39,9 \times 1,16187=46,36$ Mgr., hieraus Procent 25,698.

Rechnung $47,24 \ll \quad \ll 26,168$.

4) 20 CC. ergaben 39,9 CC. Stickstoff.

Lufttemperatur $15^{0} \mathrm{C}$. Barometerstand $754^{\mathrm{mm}}$.

1 CC. $=1,16187$ Mgr.

$39,9 \times 1,16187=46,36$ Mgr., hieraus Procent 25,698.

Rechnung $47,24 \quad \ll \quad \ll 26,186$.

5) 20 CC. ergaben 39,8 CC. Stickstoff.

Lufttemperatur $16^{\circ}$ C. Barometerstand $754^{\mathrm{mm}}$.

$1 \mathrm{CC} .=1,15657 \mathrm{Mgr}$.

$39,8 \times 1,15657=46,03 \mathrm{Mgr}$, hieraus Procent 25,515.

Rechnung $47,24 * \quad * 26,186$.

6) $20 \mathrm{CC}$ ergaben $39,8 \mathrm{CC}$. Stickstoff.

Lufttemperatur $16^{\circ} \mathrm{C}$. Barometerstand $754^{\mathrm{mm}}$.

1 CC. $=1,15657 \mathrm{Mgr}$.

$39,8 \times 1,15657=46,03 \mathrm{Mgr}$., hieraus Procent 25,515.

Rechnung $47,24 \quad \ll \quad \ll \quad$ \& 26,186 .

Die Versuche zeigen, dass in der von $200 \mathrm{CC}$. auf nur $220 \mathrm{CC}$. verdünnten Lauge eine sehr geringe, in den meisten Fällen zu vernachlässigende Absorption von Stickstoff stattfindet; sie ergeben aber weiter, dass ein mehrmaliger Gebrauch derselben Zersetzungsflüssigkeit nicht geschehen darf, da bei weiterer Verdunnung der Lauge eine nicht unerhebliche Absorption von Stickstoff , stattfindet.

Demnach würde man also für jede einzelne Bestimmung $200 \mathrm{CC}$. 
conc. Bromlange anwenden müssen, falls man die durch Stickstoffabsorption nöthig werdende Correction vermeiden wollte.

Da nun aber eine weit geringere Menge der conc. Bromlauge zur Zersetzung vollkommen ausreicht, indem 50 CC. Lange 200 Milligrm, Stickstoff entsprechen, die Anwendung eines so grossen Ueberschusses der Zersetzungsflüssigkeit ausserdem mancherlei Nachtheile, wie namentlich die unzweckmässige Grösse des Zersetzungsgefässes, die langsamere Abkühlung der beim Zersetzungsprocesse erwärmten Flüssigkeit, ferner auch einen bedeutenden Verbrauch an Brom herbeiführt, so empfiehlt es sich, eine kleinere Quantität der Lange anzuwenden und für den absorbirten Stickstoff eine Correction anzubringen.

E. Dietrich hat ebenfalls auf den durch Stickstoffabsorption entstehenden Fehler aufmerksam gemacht und hat rorgeschlagen, anstatt $200 \mathrm{CC}$. der Bromlauge nur $50 \mathrm{CC}$. zu jedem Versuch zu nehmen und den in der verdünnten Lauge gelöst bleibenden Stickstoff in Anrechnung zu bringen. Diesem Vorschlage stimme ich vollkommen bei. Ich habe auf diese Weise stets übereinstimmende und richtige Resultate erhalten. Auch die Dietrich'sche Tabelle*) für die Absorption des Stickgases habe ich für die Lösung von unterbromigsaurem Natron, obgleich sie von Dietrich für bromirte Javelle'sche Lange berechnet war, richtig gefunden, was nicht befremden kann, da das spec. Gewicht beider Laugen ein gleiches ist.

Die Grösse des Zersetzungsgefässes soll nach Knop der Menge des zu entwickelnden Stickgases ungefähr angepasst sein, d. h. wenn z. B. nar 3-5 CC. Stickstoff zu entwickeln sind, so soll das Entwickelungsgefäss ungefähr $50 \mathrm{CC}$. fassen, bei $10-12 \mathrm{CC}$. Stickstoff $200-250 \mathrm{CC}$., bei $25-80$ CC. Stickstoff $300-400$ CC., damit der im Entwickelungsgefäss bleibende Luftraum ein möglichst kleiner werde, indem durch die Erwärmung der Flüssigkeit und Luft sonst ein zu grosser Fehler entstehen könne.

Diese Raumbeschränkung halte ich für unnöthig, ich nehme stets dasselbe Entwickelungsgefäss und lasse bedeutend mehr Raum (circa 100 CC.), bringe aber die bei der Zersetzung gestiègene Temperatur des Entwickelungsgefässes durch Einstellen in kaltes Wasser genau auf denselben Temperaturgrad, den sie vor der Entwickelung hatte und lese das Volumen des Stickstoffes erst nach vollständig geschehener Abkühlung

*) Diese Zeitschrift 5, 40. 
ab. Damit die Temperatur des Kühlwassers während der Dauer eines Versuches möglichst constant bleibe, empfiehlt es sich, ein recht grosses Kühlgefäss mit schlecht leitenden Wandungen anzuwenden und nicht allzugrosse Differenzen zwischen der Temperatur der Zimmerluft und der des Kühlwassers bestehen zu lassen.

Als Gefäss zur Aufnahme der zu zersetzenden Ammoniakflüssigkeit wendet Knop ein kleines cylindrisches Gefäss- mit aufgeschliffener Glasplatte an, welches, nachdem es gefüllt und mit der Glasplatte verschlossen ist, umgekehrt and in einer Bindfadenschlinge hängend, in das die Bromlauge enthaltende Zersetzungsgefäss gelassen wird. Beim Schütteln löst sich die Glasplatte $a b$ and eine Vermischung der Flüssigkeiten findet statt. Bei nicht flüchtigen Stickstoffyerbindungen wendet $\mathrm{Knop}$ ein Zersetzungsgefäss mit verticaler Scheidewand an. Da man es aber in den allermeisten Fällen mit gebundenem Ammoniak zu thun hat, so erscheint die Anwendung eines durch ein Glasplättchen verschlossenen Cylinderchens nur in Ausnahmefällen als zweckmässig und ziehe ich dem Gebrauche einer solchen sehr unbequem zu handhabenden Vorrichtung, welche auch ein kräftiges Schütteln nicht gestattet, die auf folgende Weise herzustellende and durch die Abbildung Tafel $V$ weiter verdentlichte Einrichtung vor.

Ein kleiner $20 \mathrm{CC}$. fassender, $7,5^{\mathrm{cm}}$ hoher mit einem Ausguss versehener Cylinder wird in die Mitte des Zersetzungsgefässes gestellt, mit Gyps eingegossen und die in der Wärme getrocknete Gypsoberfläche mit einer dünnen Schicht Paraffin übergossen.

In diesen festgegossenen Cylinder wird die zu zersetzende Flüssigkeit, in dẹn äusseren weiteren Raum des Zersetzungsgefässes die Bromlauge gebracht. Die Operationen des Eingiessens, Schüttelns und Reinigens sind bei dieser Vorrichtung ausserordentlich bequem und sie gewährt den weiteren Vortheil, dass eine successive Vermischung beider Flüssigkeiten geschehen kann, wodurch einer stürmischen Entwickelung, bei welcher unzersetztes Ammoniak mit fortgerissen werden kann, vorgebeugt wird.

Die weitere Construktion des Apparates ersieht man aus der Abbildung, zu welcher nur noch folgendes kurz bemerlkt sei.

Der Hohlraum des Entwickelungsgefässes A beträgt (incl. dem des eingegossenen Cylinders) nngefähr $160 \mathrm{CC}$. Der Hals des Gefässes ist auf seiner Innenfläche mit Hülfe einer groben Feile möglichst rauh gemacht, damit jede Möglichkeit einer Verschiebung des fest und tief ein- 
zadrückenden Kautschukstopfens genommen werde. Um das Entwickelungsgefäss $\mathrm{zu}$ beschweren, damit es unter Wasser fest stehe, bringt man, bevor der kleine Cylinder eingegypst wird, einige Stücke Blei auf den Boden des, Gefässes.

Der Cylinder $B$ ist bis nahe unter dem Korke mit Wasser gefüllt, welches, um Trübung und Pilzbildung darin zu verhindern, etwas Quecksilberchlorid enthält. $\mathrm{c}$ ist eine in wirkliche Cubikcentimeter (mit 1/10 Theilung) getheilte Bürette, d. h. 1 Cubikcentimeter derselben muss dem Volumen eines Grammes Wasser von $4^{0} \mathrm{C}$. und nicht wie bei den gew öhnlichen Büretten von $17,50 \mathrm{C}$. entsprechen. ${ }^{*}$ )

Die communicirenden Röhren $c$ und $d$, sowie das Gefäss $h$ enthalten destillirtes Wasser, welches man, um den Stand der Flüssigkeit augenfälliger zu machen, mit etwas einer A nilinfarbe schwach violett färben karn. $\mathrm{e}$ ist ein Kautschukschlauch von geringer Weite und möglichst dicker Wandung.

Der Hergang der azotometrischen Stickstoffbestimmung ist nun der folgende :

In den festgegossenen kleinen Cylinder des Zersetzungsgefässes werden $10-15 \mathrm{CC}$. der zu prüfenden Ammoniaksalzlösung gebracht und mittelst eingesetztem Trichter 50 CC. Bromlauge (nach unten angegebener Vorschrift bereitet) in den weiteren Raum des Entwickelungsgefässes gegossen. Nachdem der Kautschukstopfen fest eingesetzt ist, wird das Gefäss in einen Behälter-mit kaltem Wasser (mindestens 3-4 Liter enthaltend) so tief eingesetżt, dass der Kautschukstopfen noch unter Wasser zu stehen kommt. Der Glashahn $\mathbf{f}$ wird darauf etwas gelöst, die Röhren c und $d$ durch Zusammendrücken des Kautschukballes i unter gleichzeitigem Oeffnen des Quetschhahnes $g$ mit Wasser gefüllt und durch $A b$ lassen bei $g$ der Wasserspiegel auf $U$ eingestellt. Nach etwa 5 Minuten wird der Glashahn f wieder fest eingedrückt, doch so, dass die im Gefässe $\mathrm{A}$ eingeschlossene tuft mit $\mathrm{c}$ in Communication bleibt. Man wartet 5 Minuten und beobachtet ob der Wasserspiegel in $c$ gestiegen ist; ist dies der Fall, so wird der Glashahn $\mathbf{f}$ nochmals gelüftet, wieder eingedrückt und abermals 5 Minuten gewartet. Ist der Wasserspiegel auf 0 stehen geblieben, so hat das Entwickelungsgefäss mit seinem Inhalt die Temperatur des umgebenden kalten Wassers

*) Fehlt eine solche Bürette, so kann man auch eine Currection anwenden, da die Differenz auf 100 CC. nur 0,12 CC. beträgt. 
angenommen. Man lässt nun durch Oeffnen des Quetschhahns $g$ etwa $30-40$ CC. Flüssigkeit abfliessen, nimmt das Entwickelungsgefäss aus. dem Wasser, neigt es, so dass von der zu zersetzenden Flüssigkeit ein kleiner Theil ansfliesst, dessen Vermischung mit der Bromłauge man durch Schwenken befördert, und wiederholt dies, bis der grösste Theil der ammoniakhaltigen Flüssigkeit ausgeflossen und zersetzt ist. Darauf schliesst man den Glashahn f, schüttelt kräftig um, öfnet, um den freigewordenen Stickstoff austreten zu lassen, schliesst wieder und schüttelt nochmals, bis beim Oeffnen des Glashahnes der Wasserspiegel in c nicht mehr sinkt, (ein dreimaliges kräftiges Schütteln ist gewöhnlich ausreichend), und stellt das Entwickelungsgefäss wieder in das Kühlwasser. Nach 15 Minuten hat das Entwickelungsgefäss mit seinem Inhalte wieder die frühere Temperatur (d. h. die des Kühlwassers) angenommen, während das in $c$ eingetretene Stickgas die durch das eingehängte Thermometer angezeigte Temperatur des umgebenden Wassers erhalten hat. Nachdem durch Ablassen bei $g$ der Flüssigkeitsstand in $c$ und $d$ gleichgestellt ist, wird das entwickelte. Stickstoffvolum abgelesen, ferner die Temperatur des im Cylinder B enthaltenen Wassers, sowie der-Barometerstand notirt, und unter Benutzung der Dietrich'schen Correctionstabellen, welche aus dem 5. Jahrgang dieser Zeitschrift Seite 38-40 hier nochmals auf Tafel VI abgedruckt sind, das Gewicht des Stickstoffs. berechnet.

\section{Bereitung der Bromlauge.}

$100 \mathrm{Grm}$. Aetznatron werden in Wasser gelöst und auf $1250 \mathrm{CC}$. verdünnt. Die durch Einsetzen in kaltes Wasser möglichst stark gekühlte Lauge wird mit $25 \mathrm{CC}$. Brom versetzt, kräftig geschüttelt und wiederum gekühlt. Vor Licht geschützt und gat verschlossen muss die Bromlauge aufbewahrt werden.

\section{Analytische Belege.}

I. 10,613 Grm. Chlorammonium wurden in Wasser gelöst und auf $600 \mathrm{CC}$. verdünnt.

1) 10 CC. entwickelten 38,0 CC. Stickstoff, davon blieben absorbirt 0,98 «

Totalvolum: $38,98 \mathrm{CC}$.

Temperatur 120 C., Barometerstand $758 \mathrm{~mm}$.

1 CC. Stickstoff $=1,18394 \mathrm{Mgr}$.

$38,98 \times 1,18394=46,15 \mathrm{Mgr} .$, hieraus Proc. 26,090, Rechnung $=46,32 \quad \ll \quad \ll \quad \ll 26,188$. 
Wagner: Eine Modification des Knop'schen Azotometers.

2) 10 CC. entwickelten 38,0 CC. Stickstoff, davon blieben absorbirt 0,98 «

Totalvolum: $38,98 \mathrm{CC}$.

Temperatur und Barometerstand wie bei 1, also genau das gleiche Resultat.

II. 5,424 Grm. Chlorammonium wurden in Wasser gelöst und auf 500 CC. verdünnt.

1) 15 CC. entwickelten 36,3 CC. Stickstoff, davon blieben absorbirt $0,93 \ll \quad \ll$

Totalvolum: $37,23 \mathrm{CC}$.

Temperatur $17,5^{0}$ C., Barometerstand $750 \mathrm{~mm}$.

1 CC. Stickstoff $=1,14225 \mathrm{Mgr}$.

$37,23 \times 1,14225=42,52$ Mgr., hieraus Procente 26,130. Rechnung $=42,61 \quad \ll \quad \ll \quad 26,187$.

2) 15 CC. entwickeiten 36,2 CC. Stickstoff, davon blieben absorbirt $0,93 \ll \quad \ll$.

Totalvolum: $37,13 \mathrm{CC}$.

Temperatur 170 C., Barometerstand $750 \mathrm{~mm}$.

1 CC. Stickstoff $=1,14496 \mathrm{Mgr}$.

$37,13 \times 1,14496=42,51 \mathrm{Mgr}$., hieraus Procente 26,125, Rechnong $=42,61 \quad \ll \quad \ll 20,187$.

3) 15 CC. entwickelten 36,3 CC. Stickistoff, davon blieben absorbirt $0,93 \ll \quad<$

Totalvolum: 37,23 CC.

Temperatur 17,50 C., Barometerstand $750^{\mathrm{mm}}$.

1 CC. Stickstoff $=1,14225 \mathrm{Mgr}$.

$37,23 \times 1,14225=42,52$ Mgr., hieraus Procente 26,130.

Rechnung $=42,61 \quad * \quad * 26,187$.

III. 10,0 Grm. Chlorammonium wurden in Wasser gelöst und auf 500 CC. verdünnt.

1) 10 CC. entwickelten 44,3 CC. Stickstoff, davon blieben absorbirt 1,13 «

Totalvolum : $45,43 \mathrm{CC}$.

Temperatur $15^{0}$ C., Barometerstand $748^{\mathrm{mm}}$.

1 CC. Stickstoff $=1,15247 \mathrm{Mgr}$.

$45,43 \times 1,15247=52,36 \mathrm{Mgr}$, hieraus Procente 26,178, Rechnung $=52,37 \quad * \quad * \quad * 26,188$. 
2) 10 CC. entwickelten 44,4 CC. Stickstoff, davon blieben absorhirt 1,13 «

Totalvolum: $45,53 \mathrm{CC}$.

Temperatur $15^{0}$ C., Barometerstand $748 \mathrm{~mm}$.

1 CC. Stickstoff $=1,15247 \mathrm{Mgr}$.

$45,53 \times 1,15247=52,47$ Mgr., hieraus Procente 26,235.

Rechnung $=52,37 \quad * \quad \ll \quad * 26,188$.

3) 10 CC. entwickelten 44,4 CC. Stickstoff, davon blieben absorbirt 1,13 «

Totalvolum : $45,53 \mathrm{CC}$.

Temperatur und Barometerstand wie bei 2 .

IV. 8,01 Grm. salpetersaures Ammoniak wurden in Wasser gelöst. und auf $500 \mathrm{CC}$. verdünnt.

1) 10 CC. entwickelten 23,6 CC. Stickstoff, davon blieben absorbirt $0,63 \ll$

Totalvolum: $24,23 \mathrm{CC}$.

Temperatur $17^{0}$ C., Barometerstand $756^{\mathrm{mm}}$.

1 CC. Stickstoff $=1,15429 \mathrm{Mgr}$.

$24,23 \times 1,15429=27,97$ Mgr., hieraus Procente 17,459. Rechnung $=28,03 \ll \quad \ll \quad<17,500$.

2) 15 CC. entwickelten 35,5 CC. Stickstoff, davon blieben absorbirt $0,92 \ll \quad \ll$

Totalvolum: $36,42 \mathrm{CC}$.

Temperatur $17^{0}$ C., Barometerstand $756^{\mathrm{mm}}$.

1 CC. Stickstoff $=1,15429 \mathrm{Mgr}$.

$36,42 \times 1,15429=42,04 \mathrm{Mgr}$, hieraus Procente 17,494. Rechnang $=42,05 \ll \quad \ll \quad 17,500$.

V. $10,012 \mathrm{Grm}$. schwefelsaures Ammoniak wurden in Wasser gelöst und auf $500 \mathrm{CC}$. verdünnt.

1) 10 CC. entwickelten 35,6 CC. Stickstoff, davon blieben absorbirt 0,93 « $\quad \ll$

Totalvolum : $36,53 \mathrm{CC}$.

Temperatur $15^{0}$ C., Barometerstand $754^{\mathrm{mm}}$.

1 CC. Stickstoff $=1,16187$ Mgr.

$36,53 \times 1,16187=42,44$ Mgr., hieraus Procente 21,194 . R̀echnung $=42,475 * \quad \ll \quad 21,212$. 
2) 10 CC. entwickelten 35,8 CC. Stickstoff, davon blieben absorbirt $0,93 \ll \quad$ «

Totalvolum: $36,73 \mathrm{CC}$.

Temperatur $16^{0}$ C., Barometerstand $754^{\mathrm{mm}}$.

1 CC. Stickstofi $=1,15657 \mathrm{Mgr}$.

$36,73 \times 1,15657=42,48 \mathrm{Mgr}$., hieraus Procente 21,214 .

Rechnung $=42,475 \quad \ll \quad \ll \quad 21,212$.

3) 10 CC. entwickelten 35,7 CC. Stickstoff,

davon blieben absorbirt $0,93 \ll<$

Totalvolum: $36,63 \mathrm{CC}$.

Temperatur $16^{\circ}$ C., Barometerstand $754^{\mathrm{mm}}$.

1 CC. Stickstoff $=1,15657 \mathrm{Mgr}$.

$36,63 \times 1,15657=42,36 \mathrm{Mgr}$., hieraus Procente 21,154.

Rechnung $=42,475 * \ll \quad \ll \quad 21,212$.

Die Resultate der azotometrischen Bestimmungen fallen fast a b sol u t genau aus, wie dies schon von Dietrich gezeigt worden ist. Die Ausführung der Bestimmungen ist zugleich so ausserordentlich einfach und schnell, dass diese Methode in der That kaum etwas zu wünschen übrig lässt.

Für agriculturchemische Zwecke hat der Azotometer einen ganz besonderen Werth. Bei der Untersuchung der Ammoniak - Düngemittel z. B. leistet dasselbe vortreffliche Dienste, indem einfach die Lösung des Ammoniaksalzes oder der wässerige Auszug des Düngemittels in das Zersetzungsgefäss gebracht wird, und innerhalb kurzer Zeit mehrere Controlbestimmungen ausgeführt werden können.

Selbstrerständlich lässt sich auf diese Weise nur der in Form von Ammoniak vorhandene Stickstoff bestimmen. Sobald der in den Düngemitteln enthaltene Stickstoff nur zum Theil aus Ammoniaksalzen besteht, müssen andere Methoden angewendet werden, um den Gehalt an GesammtStickstoff festzustellen. In Rücksicht auf den ungleichen Düngewerth der verschiedenen Stickstoffverbindungen bleibt es jedoch immerhin von grossem Werth, in solchen Düngemitteln den in Ammoniakform vorhandenen Stickstoff auf leichte Weise für sich bestimmen zu können.

Hat man es endlich mit Substanzen zu thun, welche bei der Verbrennung mit Natronkalk die vorgelegte Säure sehr dunkel färben, so empfiehlt es sich, die Varrentrapp-Will'sche Methode mit der 
azotometrischen Stickstoffbestimmung zu combiniren. Schliesslich noch die Bemerkung, dass die Handlung Ehrhardt \& Metzger in Darmstadt den oben beschriebenen Apparat anfertigen lassen and zum Verkauf vorräthig halten wird.

Ueber selenigsaure Magnesia.

Von

\section{Prof. Dr. Hilger.}

Bezugnehmend auf meine Mittheilangen in Jahrgang XIII. Heft 2. dieser Zeitschrift theile ich nachstehend Näheres über die Constitutionsverhältnisse jener Fällung mit, welche entsteht, wenn Lösungen seleniger Säure mit Chlormagnesium und Ammoniak bei Gegenwart von Chlorammonium versetzt werden. Die krystallinische Fällung, welche stets nach längerem Stehen sich erst vollständig bildet, enthält, wie früher erwähnt: Selenige Säure, Magnesium und Ammoniak, letzteres jedoch in stets wechselnden Mengen, nie fehlend, auch weun wiederholt ansgewaschen wurde.

Herr Dr. v. Gerichten hat die quantitative Bestimmung ausgeführt und es resultirten folgende Verhältnisse:

\begin{tabular}{|c|c|c|c|}
\hline \multirow[b]{3}{*}{$\mathrm{Se}_{2}$} & \multicolumn{2}{|c|}{ Gefunden. } & Berechnet \\
\hline & I. & II. & auf $\operatorname{se}_{3} \mathrm{Mg}+7 \mathrm{H}_{2} \theta$ \\
\hline & - 40,60 & 40,76 & 40,28 \\
\hline $\operatorname{Mg} \theta$ & 13,91 & $\mathbf{1 4 , 4 1}$ & 14,38 \\
\hline$\left(\mathrm{NH}_{4}\right)_{2} \theta$ & - & 0,46 & - \\
\hline $\mathrm{H}_{2} \theta$. & - & - & 45,32 \\
\hline
\end{tabular}

Im ersten Falle wurde $\$ \mathrm{~B}_{2}$ als $\mathrm{Se}$ bestimmt, im zweiten aus $\mathrm{Se} \mathrm{S}_{2}$ berechnet.

Die vorliegenden Zahlen berechtigen unbedingt z̧ur Formel

$$
\mathrm{SeO}_{3} \mathrm{Mg}, 7 \mathrm{H}_{2} \mathrm{O}
$$

ohne Ammoniakgehalt, der sicher nur mechanisch eingeschlossen ist.

Berzelius beschrieb schon früher eine selenigsaure Magnesia mit $3 \mathrm{H}_{2} \theta$, durch Neutralisation von seleniger Säure mit kohlensaurer Magnesia erhalten.

Die krystallinische Beschaffenheit dieser Verbindung zeigte sich, wie bereits erwähnt, stets in Uebereinstimmung mit der phosphorsauren 